\title{
There is a slight increase in incident diabetes risk with the use of statins, but benefits likely outweigh any adverse effects in those with moderate-to-high cardiovascular risk
}

\section{Lokpal Bhatia, Christopher D Byrne}

\author{
Commentary on: Sattar N, Preiss D, Murray HM, et al. Statins and risk of incident diabetes: a \\ collaborative meta-analysis of randomised statin trials. Lancet 2010;375:735-42.
}

The Institute of Developmental Sciences, University of Southampton, Southampton, UK

\section{Correspondence to:} Christopher D Byrne The Institute of Developmental Sciences (IDS Building), University of Southampton, MP 887, Southampton General Hospital, Tremona Rd, Southampton S016 6YD, UK; cdtb@soton.ac.uk
Statins are one of the most widely used drug classes, with approximately 50 million prescriptions dispensed in England alone in 2008. Their efficacy and safety in significantly reducing cardiovascular events in moderateto-high-risk patients has been well documented, both in primary and secondary prevention. ${ }^{1}$ Commonly reported side-effects include muscle aches and increases in liver enzymes, but, in general, statins are well tolerated with a low incidence of side-effects. However, the recent collaborative meta-analysis of 13 major placebo-controlled statin trials by Sattar and colleagues reports a $9 \%$ increased risk for incident diabetes over 4 years (OR 1.09; 95\% CI
1.02-1-17) in patients randomised to statins compared to those assigned to placebo. Heterogeneity between trials was low $\left(I^{2}=11 \%\right)$, suggesting that this risk appears to be a true class effect, despite known differences in lipophilicity and metabolic clearance pathways between individual statin drugs.

Although these findings are of concern, it is worth pointing out that all meta-analyses have inherent limitations. Importantly, as acknowledged by Sattar and colleagues, there was inter- and intra-trial variability of the methods used to define a diagnosis of incident diabetes, with some trials employing a physician-reported diagno- 
sis and others utilising fasting blood glucose measurements. When the authors analysed the trials using only fasting glucose concentrations, the difference between statin and placebo groups was not statistically significant, which the authors suggest may have been due to a type 2 statistical error attributable to limited power. Unfortunately, there were no available data on differences in baseline fasting glucose concentrations or family history of diabetes between randomisation groups, changes in body mass index from baseline to follow up, use of diabetogenic drugs or levels of blood pressure control within trials. Each of these factors could have influenced risk of incident diabetes and have affected heterogeneity between trials, and, in turn, potentially confounding the findings, although in our opinion this is unlikely given that trials were randomised. It is noteworthy that in half of the analysed trials, incident diabetes was reported as a post hoc finding, rather than as a prespecified end point. However, despite these limitations, the large numbers of patients analysed give good statistical power to the metaanalysis; thus, the consistent finding of statin-induced diabetes between trials cannot be dismissed, suggesting that the finding is real and cannot be explained away by attributing it to chance, bias or any confounding effects.

Given that diabetes increases the risk of cardiovascular mortality by two- to four-fold, we are potentially presented with a paradox of statin benefit versus harm. It is worth noting that the absolute risk of diabetes documented within the meta-analysis is indeed very low; that is, treatment with a statin for 1000 patient years resulted in only one excess case of diabetes. Comparing to other cardiovascular drugs known to increase the risk of diabetes (eg, beta-blockers and thiazide diuretics), statins are 3 times less likely to cause diabetes. ${ }^{2}$ Importantly, statins are approximately 8 times more likely to prevent a cardiovascular event than cause a case of incident diabetes and, therefore, the risk-benefit ratio is undoubtedly still hugely in favour of statin therapy for at-risk groups.

How might statins influence glucose tolerance and cause diabetes? Possible mechanisms include (a) reduction in metabolites produced during cholesterol synthesis, which would normally upregulate Glut-4, and (b) indirect inhibition of pancreatic insulin release. ${ }^{3}$ Interestingly, although there is a well-known positive correlation between inflammation and insulin resistance, high-dose statins worsened glycaemic control, despite reducing high-sensitivity CRP to a greater extent than standarddose statins. ${ }^{4}$ This dissociation between statin-induced improvements in markers of inflammation and statininduced changes in glucose tolerance was also evident in the JUPITER trial of rosuvastatin, which suggests that the association between statins and incident diabetes represents a complex interplay of molecular, genetic and physiological factors that requires further understanding. Although some of the mechanisms responsible for affecting glucose concentrations may be due to differing lipophilicity of statins, the degree of lipophilicity does not mediate the clinical effectiveness of statins to decrease risk of myocardial infarction and stroke. Moreover, largescale clinical trials have not shown any differential effects between hydrophilic (eg, pravastatin) and more lipophilic (eg, simvastatin, atorvastatin) statins on cardiovascular end points.

Should this meta-analysis alter our practice of statin prescribing? The simple answer is no, when considering individuals at moderate or high cardiovascular risk, or with documented disease. However, it should encourage us to be more vigilant in identifying patients at high risk of developing diabetes (eg, individuals with the metabolic syndrome), when assessing the risk-benefit ratio of statin use in patients with only modest cardiovascular risk. It also emphasises the need to continue to recommend/support lifestyle change and monitor plasma glucose concentrations in patients on long-term statins. Any future statin trials should include incident diabetes or impaired glucose regulation as a prespecified secondary end point.

Competing interests $\mathrm{CDB}$ has receiving funding in the form of an Independent Research Grant from Pfizer.

\section{References}

1. Baigent C, Keech A, Kearney PM, et al. Efficacy and safety of cholesterol-lowering treatment: prospective meta-analysis of data from 90,056 participants in 14 randomised trials of statins. Lancet 2005;366:1267-78.

2. Elliott WJ, Meyer PM. Incident diabetes in clinical trials of antihypertensive drugs: a network meta-analysis. Lancet 2007;369:201-7.

3. Sasaki J, Iwashita M, Kono S. Statins: beneficial or adverse for glucose metabolism. J Atheroscler Thromb 2006;13:123-9.

4. Sabatine MS, Wiviott SD, Morrow DA, Mccabe CH, Cannon CP. High-dose atorvastatin associated with worse glycaemic control: a PROVE-IT TIMI 22 substudy. Circulation 2004;110:834. 